SELECCIONES MATEMÁTICAS
Universidad Nacional de Trujillo
ISSN: $2411-1783$ (Online)
Vol. 06(01): $41-48(2019)$

\title{
Algunas desigualdades integrales fraccionarias del tipo Hermite Hadamard y Minkowski.
}

\section{Some fractional integral inequalities of Hermite Hadamard and Minkowski type. Jorge Eliecer Hernádez Hernández *}

DOI: http://dx.doi.org/10.17268/sel.mat.2019.01.07

\begin{abstract}
Resumen
En este artículo se establecen algunas desigualdades integrales fraccionarias del tipo Hermite-Hadamard y Minkowski haciendo uso del operador integral fraccionario definido por R.K. Raina (2016) en [1], las cuales generalizan algunos resultados previos encontrados por L. Bougoffa [5] y S.S. Dragomir [7].
\end{abstract}

Palabras clave. Desigualdades integrales - Operador integral fraccionario.

\begin{abstract}
This article presents some fractional integral inequalities of the Hermite-Hadamard and Minkowski type using the fractional integral operator defined by R.K. Raina (2016) in [1], which generalize some previous results found by L. Bougoffa [5] and S.S. Dragomir [7].
\end{abstract}

Keywords. Integral inequalities - Fractional integral operator

1. Introducción. Innumerables trabajos de investigación en los cuales el tema de las desigualdades es recurrente debido a que las mismas juegan un papel significativo en todos los campos de las Matemáticas y ciencias en general. La publicación del libro Inequalities de H.G.Hardy, J. E. Littlewood and G. Polya [10] se convierte en una fuente de referencia para muchos investigadores, lo mismo que las publicaciones de E. Bechenbach y $R$. Bellman [2, 3]. Como ejemplo, las desigualdades integrales, incluyendo aquellas de tipo fraccionario, han sido estudiadas en diversas investigaciones [4, 13, 14]. La desigualdad de Hermite-Hadamard:

$$
f\left(\frac{a+b}{2}\right) \leq \frac{1}{(b-a)} \int_{a}^{b} f(x) d x \leq \frac{f(a)+f(b)}{2}
$$

donde f es una función convexa, es una que ha recibido especial antención en los últimos años [6, 12, 18].

Su origen data de los trabajos de Charles Hermite [11] y Jacques Hadamard [9], como lo expone la recopilación histórica respecto a la misma hecha por C.P. Niculescu and L.E. Persson [16].

A finales del siglo XIX con los trabajos de Riemann y Liouville, aparece lo que hoy se denomina Cálculo Fraccional, el cual obtuvo un gran desarrollo y encontró diversos campos de aplicación en ciencias física, ingeniería, ciencias biológicas y económicas [8, 15]. Las desigualdades que involucran operadores integrales fraccionarios también se han estudiado en $[1,12,18]$.

Dado que el trabajo en esta dirección ha recibido mucha atención, se pretende establecer una formulación general en este artículo y sus correspondientes implicaciones produzcan ciertas desigualdades nuevas.

El objetivo del presente trabajo es establecer algunas desigualdades integrales fraccionarias para funciones no negativas e integrables, las cuales están relacionadas con la desigualdad de Hermite-Hadamard usando el

*ID ORCID: http://orcid.org/0000-0002-4406-5469, Universidad Centroccidental Lisandro Alvarado, Decanato de Ciencias Económicas y Empresariales, Av.20 esquina Av. Moran, Barquisimeto, Venezuela. (jorgehernandez@ucla.edu.ve). 
operador integral fraccionario definido por R.K. Raina. Además se establecen otras del tipo Minkowski. Los resultados obtenidos generalizan otros ya establecidos en los trabajos de L. Bougoffa [5] y S.S. Dragomir [7].

\section{Preliminares.}

En esta sección se presentan algunas definiciones y propiedades que serán usadas a lo largo del desarrollo de este trabajo.

Definición 1. Una función real $f(t)$ definida para $t>0$ pertenece al espacio $C_{\mu}, \mu \in \mathbb{R}$ si existe un número real $p>\mu$ tal que $f(t)=t^{p} f_{1}(t)$, donde $f_{1} \in C([0, \infty))$.

Definición 2. Una función real $f(t)$ definida para $t>0$, pertenece al espacio $C_{\mu}^{(n)}, \mu \in \mathbb{R}$ si $f^{(n)} \in C_{\mu}$.

En [17], R. K. Raina definió, formalmente, una clase de funciones por medio de

$$
\mathcal{F}_{\rho, \lambda}^{\sigma}(x)=\mathcal{F}_{\rho, \lambda}^{\sigma(0), \sigma(1), . .}(x)=\sum_{k=0}^{\infty} \frac{\sigma(k)}{\Gamma(\rho k+\lambda)} x^{k}
$$

donde $\rho, \lambda>0,|x| \in \mathbb{R} y \sigma=(\sigma(1), . ., \sigma(k), .$.$) es una sucesión acotada de números reales.$

Usando (2.1), R.P. Agarwal, M-J Luo and R.K Raina, en [1], definieron el operador integral fraccionario izquierdo y derecho, respectivamente, de la siguiente manera:

$$
\left(\mathcal{J}_{\rho, \lambda, a+; w}^{\sigma} \varphi\right)(x)=\int_{a}^{x}(x-t)^{\lambda-1} \mathcal{F}_{\rho, \lambda}^{\sigma}\left[w(x-t)^{\rho}\right] \varphi(t) d t, \quad(x>a)
$$

$$
\left(\mathcal{J}_{\rho, \lambda, b-; w}^{\sigma} \varphi\right)(x)=\int_{x}^{b}(t-x)^{\lambda-1} \mathcal{F}_{\rho, \lambda}^{\sigma}\left[w(t-x)^{\rho}\right] \varphi(t) d t, \quad(x<b),
$$

donde $\lambda, \rho>0, w \in \mathbb{R}$ y $\varphi$ es una función tal que la integral expuesta en (2.2) y (2.3) existen.

Es fácil verificar que $\mathcal{J}_{\rho, \lambda, a+; w}^{\sigma} y \mathcal{J}_{\rho, \lambda, b-; w}^{\sigma}$ son operadores integrales acotados sobre $L(a, b)$, si

$$
\mathfrak{M}:=\mathcal{F}_{\rho, \lambda+1}^{\sigma}\left[w(b-a)^{\rho}\right]<\infty .
$$

En efecto, para $\varphi \in L((a, b))$ se tiene que

$$
\left\|\mathcal{J}_{\rho, \lambda, a+; w}^{\sigma} \varphi\right\|_{1} \leq \mathfrak{M}\|\varphi\|_{1}
$$

$y$

$$
\left\|\mathcal{J}_{\rho, \lambda, b-; w}^{\sigma} \varphi\right\|_{1} \leq \mathfrak{M}\|\varphi\|_{1}
$$

donde

$$
\|\varphi\|_{1}=\int_{a}^{b}|\varphi(x)| d x .
$$

La importancia de estos operadores radica precisamente en su generalidad. Muchos, y útiles, operadores integrales fraccionarios pueden ser obtenidos a partir de una escogencia específica de los coeficientes de la suceción $\sigma$. Por ejemplo, los operadores integrales fraccionarios (izquierdo y derecho) de Riemann-Liouville, $I_{a+}^{\alpha}$ y $I_{b-}^{\alpha}$ de orden $\alpha$ :

$$
\left(I_{a+}^{\alpha} \varphi\right)(x)=\frac{1}{\Gamma(\alpha)} \int_{a}^{x}(x-t)^{\alpha-1} \varphi(t) d t, \quad(x>a)
$$

$y$

$$
\left(I_{b-}^{\alpha} \varphi\right)(x)=\frac{1}{\Gamma(\alpha)} \int_{x}^{b}(t-x)^{\alpha-1} \varphi(t) d t, \quad(x<b)
$$

se encuentran a partir de (2.2) y (2.3) haciendo $\lambda=\alpha, \sigma(0)=1$ y $w=0$.

El siguiente Lema es de utilidad para el desarrollo del presente trabajo; su demostración se encuentra en [6].

Lema 1. Sea h una función real concava sobre el intervalo $[a, b]$. Si $x \in[a, b]$ entonces

$$
h(a)+h(b) \leq h(b+a-x)+h(x) \leq 2 h\left(\frac{a+b}{2}\right) .
$$




\section{Resultados Obtenidos.}

Teorema 1. Sean $\lambda, \rho>0, p \geq 1, w \in \mathbb{R} y \sigma=\{\sigma(k)\}_{k=0}^{\infty}$ una sucesión acotada de números reales positivos. Sean $f, g$ funciones reales definidas sobre $[a, \infty)$, tal que $\mathcal{J}_{\rho, \lambda, a+; w}^{\sigma} f^{p}(x)<\infty$ y $\mathcal{J}_{\rho, \lambda, a+; w}^{\sigma} g^{p}(x)<\infty$. Si $0<m \leq(f(t) / g(t)) \leq M, t \in[a, x]$, entonces se tiene la siguiente desigualdad

$$
\left[\left(\mathcal{J}_{\rho, \lambda, a+; w}^{\sigma} f^{p}\right)(x)\right]^{1 / p}+\left[\left(\mathcal{J}_{\rho, \lambda, a+; w}^{\sigma} g^{p}\right)(x)\right]^{1 / p} \leq\left(\frac{1+M(m+2)}{(M+1)(m+1)}\right)\left[\left(\mathcal{J}_{\rho, \lambda, a+; w}^{\sigma}(f+g)^{p}\right)(x)\right]^{1 / p}
$$

Demostración: Como $f(t) / g(t)<M, t \in[a, x], x>0$ entonces se tiene que

$$
f(t) \leq M g(t)+M f(t)-M f(t)
$$

y así

$$
(M+1)^{p} f^{p}(t) \leq M^{p}(f+g)^{p}(t) .
$$

Multiplicando ambos lados por $(x-t)^{\lambda-1} \mathcal{F}_{\rho, \lambda}^{\sigma}\left[w(x-t)^{\rho}\right]$ se obtiene que

$$
(x-t)^{\lambda-1} \mathcal{F}_{\rho, \lambda}^{\sigma}\left[w(x-t)^{\rho}\right](M+1)^{p} f^{p}(t) \leq(x-t)^{\lambda-1} \mathcal{F}_{\rho, \lambda}^{\sigma}\left[w(x-t)^{\rho}\right] M^{p}(f+g)^{p}(t) .
$$

Integrando respecto a $t \in[a, x]$ se encuentra que

$$
(M+1)^{p} \int_{a}^{x}(x-t)^{\lambda-1} \mathcal{F}_{\rho, \lambda}^{\sigma}\left[w(x-t)^{\rho}\right] f^{p}(t) d t \leq M^{p} \int_{a}^{x}(x-t)^{\lambda-1} \mathcal{F}_{\rho, \lambda}^{\sigma}\left[w(x-t)^{\rho}\right](f+g)^{p}(t) d t
$$

lo cual es equivalente a

$$
\left(\mathcal{J}_{\rho, \lambda, a+; w}^{\sigma} f^{p}\right)(x) \leq \frac{M^{p}}{(M+1)^{p}}\left(\mathcal{J}_{\rho, \lambda, a+; w}^{\sigma}(f+g)^{p}\right)(x)
$$

por lo tanto

$$
\left[\left(\mathcal{J}_{\rho, \lambda, a+; w}^{\sigma} f^{p}\right)(x)\right]^{1 / p} \leq \frac{M}{(M+1)}\left[\left(\mathcal{J}_{\rho, \lambda, a+; w}^{\sigma}(f+g)^{p}\right)(x)\right]^{1 / p}
$$

Ahora, usando el hecho que $m g(t) \leq f(t)$, se obtiene

$$
\left(1+\frac{1}{m}\right) g(t) \leq \frac{1}{m}(f+g)(t)
$$

y similarmente se encuentra que

$$
\left[\left(\mathcal{J}_{\rho, \lambda, a+; w}^{\sigma} g^{p}\right)(x)\right]^{1 / p} \leq \frac{1}{(m+1)}\left[\left(\mathcal{J}_{\rho, \lambda, a+; w}^{\sigma}(f+g)^{p}\right)(x)\right]^{1 / p} .
$$

Sumando (3.2) y (3.3) se obtiene la desigualdad buscada (3.1).

Observación 1.

Haciendo $\lambda=\alpha, \sigma=(1,0,0,0, \ldots), w=0$ y $a=0$ en el Teorema 1 se tiene que

$$
\left[\left(I_{0+}^{\alpha} f^{p}\right)(x)\right]^{1 / p}+\left[\left(I_{0+}^{\alpha} g^{p}\right)(x)\right]^{1 / p} \leq\left(\frac{1+M(m+2)}{(M+1)(m+1)}\right)\left[\left(I_{0+}^{\alpha}(f+g)^{p}\right)(x)\right]^{1 / p}
$$

lo cual coincide con el Teorema 2.1 en [6]. En particular, si $\alpha=1$ entonces

$$
\|f\|_{p}+\|g\|_{p} \leq\left(\frac{1+M(m+2)}{(M+1)(m+1)}\right)\|f+g\|_{p},
$$

haciendo coincidencia con el Teorema 1.2 en [5].

Teorema 2. Sean $p \geq 1, \lambda, \rho>0, w \in R y \sigma=(\sigma(k))_{k=0}^{\infty}$ una sucesión acotada de números reales positivos. Sean $f, g$ funciones reales definidas sobre $[a, \infty)$, tales que $\left(\mathcal{J}_{\rho, \lambda, a+; w}^{\sigma} f^{p}\right)(x)<\infty y\left(\mathcal{J}_{\rho, \lambda, a+; w}^{\sigma} g^{p}\right)(x)<\infty$. Si $0<m \leq(f(t) / g(t)) \leq M, t \in[a, x]$, entonces se tiene que

$$
\left[\left(\mathcal{J}_{\rho, \lambda, a+; w}^{\sigma} f^{p}\right)(x)\right]^{2 / p}+\left[\left(\mathcal{J}_{\rho, \lambda, a+; w}^{\sigma} g^{p}\right)(x)\right]^{2 / p}
$$




$$
\geq\left(\frac{(M+1)(m+1)}{M}-2\right)\left[\left(\mathcal{J}_{\rho, \lambda, a+; w}^{\sigma} f^{p}\right)(x)\right]^{1 / p}\left[\left(\mathcal{J}_{\rho, \lambda, a+; w}^{\sigma} g^{p}\right)(x)\right]^{1 / p} .
$$

Demostración: Multiplicando (3.2) y (3.3) sigue que

$$
\frac{(M+1)(m+1)}{M}\left[\left(\mathcal{J}_{\rho, \lambda, a+; w}^{\sigma} f^{p}\right)(x)\right]^{1 / p}\left[\left(\mathcal{J}_{\rho, \lambda, a+; w}^{\sigma} g^{p}\right)(x)\right]^{1 / p} \leq\left[\left(\mathcal{J}_{\rho, \lambda, a+; w}^{\sigma}(f+g)^{p}\right)(x)\right]^{2 / p} .
$$

Aplicando la desigualdad de Minkowski en la parte derecha de (3.4), se tiene que

$$
\left[\left(\mathcal{J}_{\rho, \lambda, a+; w}^{\sigma}(f+g)^{p}\right)(x)\right]^{2 / p} \leq\left(\left[\left(\mathcal{J}_{\rho, \lambda, a+; w}^{\sigma} f^{p}\right)(x)\right]^{1 / p}+\left[\left(\mathcal{J}_{\rho, \lambda, a+; w}^{\sigma} g^{p}\right)(x)\right]^{1 / p}\right)^{2} .
$$

Sigue que

$$
\begin{aligned}
& {\left[\left(\mathcal{J}_{\rho, \lambda, a+; w}^{\sigma}(f+g)^{p}\right)(x)\right]^{2 / p}} \\
& \qquad\left[\left(\mathcal{J}_{\rho, \lambda, a+; w}^{\sigma} f^{p}\right)(x)\right]^{2 / p}+\left[\left(\mathcal{J}_{\rho, \lambda, a+; w}^{\sigma} g^{p}\right)(x)\right]^{2 / p} \\
& \quad+2\left[\left(\mathcal{J}_{\rho, \lambda, a+; w}^{\sigma} f^{p}\right)(x)\right]^{1 / p}\left[\left(\mathcal{J}_{\rho, \lambda, a+; w}^{\sigma} g^{p}\right)(x)\right] . .^{1 / p}
\end{aligned}
$$

Usando las desigualdades (3.4) y (3.5) se encuentra el resultado buscado.

\section{Observación 2.}

Haciendo $\lambda=\alpha, \sigma=(1,0,0, \ldots), w=0$ y $a=0$ en el Teorema 2 se tiene que

$$
\left[\left(I_{0+}^{\alpha} f^{p}\right)(x)\right]^{2 / p}+\left[\left(I_{0+}^{\alpha} g^{p}\right)(x)\right]^{2 / p} \geq\left(\frac{(M+1)(m+1)}{M}-2\right)\left[\left(I_{0+}^{\alpha} f^{p}\right)(x)\right]^{1 / p}\left[\left(I_{0+}^{\alpha} g^{p}\right)(x)\right]^{1 / p}
$$

la cual coincide con el Teorema 2.3 en [6]. En particular, si $\alpha=1$ entonces

$$
\|f\|_{p}^{2}+\|g\|_{p}^{2} \geq\left(\frac{(M+1)(m+1)}{M}-2\right)\|f\|_{p}\|g\|_{p}
$$

haciendo coincidencia con el Teorema 2.2 en [7].

Teorema 3. Sean $p, q>1, \lambda_{1}, \lambda_{2}, \rho>0, w \in \mathbb{R} y \sigma=\{\sigma(k)\}_{k=0}^{\infty}$ una sucesión acotada de números reales positivos. Sean $f, g$ funciones de valor real, positivas, definidas en $[0, \infty)$. Si $f^{p}$ y $g^{q}$ son cóncavas en $[0, \infty)$, se tiene que

$$
\begin{aligned}
2^{-p-q}(f(0)+f(x))^{p} & (g(0)+g(x))^{q}\left(\mathcal{J}_{\rho, \lambda_{2}, 0+; w}^{\sigma}\left(x^{\lambda_{1}-1}\right)\right)^{2} \\
\leq & \left(\mathcal{J}_{\rho, \lambda_{2}, 0+; w}^{\sigma}\left(x^{\lambda_{1}-1} f^{p}\right)\right)(x)\left(\mathcal{J}_{\rho, \lambda_{2}, 0+; w}^{\sigma}\left(x^{\lambda_{1}-1} g^{q}\right)\right)(x) .
\end{aligned}
$$

Demostración: Como las funciones $f^{p}$ y $g^{q}$ son cóncavas en $[0, \infty)$, usando el Lema 1 , para cualquier $x \in$ $[0, \infty)$ se tiene que

$$
f^{p}(0)+f^{p}(x) \leq f^{p}(x-t)+f^{p}(t) \leq 2 f^{p}\left(\frac{x}{2}\right)
$$

and

$$
g^{p}(0)+g^{p}(x) \leq g^{p}(x-t)+g^{p}(t) \leq 2 g^{p}\left(\frac{x}{2}\right)
$$

Multiplicando (3.6) y (3.7) por $(x-t)^{\lambda_{1}-1}(t)^{\lambda_{2}-1} \mathcal{F}_{\rho, \lambda_{2}}^{\sigma}\left[w t^{\rho}\right]$ se obtiene

$$
\left(f^{p}(0)+f^{p}(x)\right) \int_{0}^{x}(x-t)^{\lambda_{1}-1}(t)^{\lambda_{2}-1} \mathcal{F}_{\rho, \lambda_{2}}^{\sigma}\left[w t^{\rho}\right] d t
$$




$$
\begin{gathered}
\leq \int_{0}^{x}(x-t)^{\lambda_{1}-1}(t)^{\lambda_{2}-1} \mathcal{F}_{\rho, \lambda_{2}}^{\sigma}\left[w t^{\rho}\right] f^{p}(x-t) d t \\
+\int_{0}^{x}(x-t)^{\lambda_{1}-1}(t)^{\lambda_{2}-1} \mathcal{F}_{\rho, \lambda_{2}}^{\sigma}\left[w t^{\rho}\right] f^{p}(t) d t \\
\leq 2 f^{p}\left(\frac{x}{2}\right) \int_{0}^{x}(x-t)^{\lambda_{1}-1}(t)^{\lambda_{2}-1} \mathcal{F}_{\rho, \lambda_{2}}^{\sigma}\left[w t^{\rho}\right] d t
\end{gathered}
$$

y

$$
\begin{aligned}
& \left(g^{q}(0)+g^{q}(x)\right) \int_{0}^{x}(x-t)^{\lambda_{1}-1}(t)^{\lambda_{2}-1} \mathcal{F}_{\rho, \lambda_{2}}^{\sigma}\left[w t^{\rho}\right] d t \\
& \leq \int_{0}^{x}(x-t)^{\lambda_{1}-1}(t)^{\lambda_{2}-1} \mathcal{F}_{\rho, \lambda_{2}}^{\sigma}\left[w t^{\rho}\right] g^{q}(x-t) d t \\
& \quad+\int_{a}^{x}(x-t)^{\lambda_{1}-1}(t)^{\lambda_{2}-1} \mathcal{F}_{\rho, \lambda_{2}}^{\sigma}\left[w t^{\rho}\right] g^{q}(t) d t \\
& \leq 2 g^{q}\left(\frac{x}{2}\right) \int_{a}^{x}(x-t)^{\lambda_{1}-1}(t)^{\lambda_{2}-1} \mathcal{F}_{\rho, \lambda_{2}}^{\sigma}\left[w t^{\rho}\right] d t .
\end{aligned}
$$

Usando el cambio de variable $x-t=y$ se tiene que

$$
\begin{aligned}
\int_{0}^{x}(x- & t)^{\lambda_{1}-1}(t)^{\lambda_{2}-1} \mathcal{F}_{\rho, \lambda_{2}}^{\sigma}\left[w(t)^{\rho}\right] f^{p}(x-t) d t \\
& =\int_{0}^{x}(y)^{\lambda_{1}-1}(x-y)^{\lambda_{2}-1} \mathcal{F}_{\rho, \lambda_{2}}^{\sigma}\left[w(x-y)^{\rho}\right] f^{p}(y) d y \\
& =\left(\mathcal{J}_{\rho, \lambda_{1}, 0+; w}^{\sigma} x^{\lambda_{1}-1} f^{p}\right)(x)
\end{aligned}
$$

y

$$
\begin{aligned}
\int_{a}^{x}(x-t)^{\lambda_{1}-1}(t)^{\lambda_{2}-1} \mathcal{F}_{\rho, \lambda_{2}}^{\sigma}\left[w(t)^{\rho}\right] g^{q}(x-t) d t \\
\quad=\int_{a}^{x}(y)^{\lambda_{1}-1}(x-y)^{\lambda_{2}-1} \mathcal{F}_{\rho, \lambda_{2}}^{\sigma}\left[w(x-y)^{\rho}\right] g^{q}(y) d y \\
=\left(\mathcal{J}_{\rho, \lambda_{2}, 0+; w}^{\sigma} x^{\lambda-1} g^{q}\right)(x)
\end{aligned}
$$

\section{Entonces,}

$$
\begin{aligned}
\left(f^{p}(0)+f^{p}(x)\right)\left(\mathcal{J}_{\rho, \lambda_{2}, 0+; w}^{\sigma} x^{\lambda_{1}-1}\right) & \leq 2\left(\mathcal{J}_{\rho, \lambda_{2}, 0+; w}^{\sigma} x^{\lambda_{1}-1} f^{p}\right)(x) \\
& \leq 2 f^{p}\left(\frac{x}{2}\right)\left(\mathcal{J}_{\rho, \lambda_{2}, 0+; w}^{\sigma} x^{\lambda_{1}-1}\right)
\end{aligned}
$$

Similarly, for $g$ we have

$$
\begin{aligned}
\left(g^{q}(0)+g^{q}(x)\right)\left(\mathcal{J}_{\rho, \lambda_{2}, 0+; w}^{\sigma} x^{\lambda_{1}-1}\right) & \leq 2\left(\mathcal{J}_{\rho, \lambda_{2}, 0+; w}^{\sigma} x^{\lambda_{1}-1} g^{q}\right)(x) \\
& \leq 2 g^{q}\left(\frac{x}{2}\right)\left(\mathcal{J}_{\rho, \lambda_{2}, 0+; w}^{\sigma} x^{\lambda_{1}-1}\right) .
\end{aligned}
$$

Las desigualdades (3.8) y (3.9) implican que

$$
\begin{aligned}
\left(f^{p}(0)+f^{p}(x)\right)\left(g^{q}(0)\right. & \left.+g^{q}(x)\right)\left(\mathcal{J}_{\rho, \lambda_{2}, 0+; w}^{\sigma} x^{\lambda_{1}-1}\right)^{2} \\
\leq & 4\left(\mathcal{J}_{\rho, \lambda, 0+; w}^{\sigma} x^{\lambda-1} f^{p}\right)(x)\left(\mathcal{J}_{\rho, \lambda_{2}, 0+; w}^{\sigma} x^{\lambda_{1}-1} g^{q}\right)(x) .
\end{aligned}
$$


Por otra parte, como $f$ y $g$ son funciones positivas, entonces para cualquier $x>0, p \geq 1, q \geq 1$ se tiene que

$$
\left(\frac{f^{p}(0)+f^{p}(x)}{2}\right)^{1 / p} \geq \frac{f(0)+f(x)}{2}
$$

y

$$
\left(\frac{g^{q}(0)+g^{q}(x)}{2}\right)^{1 / p} \geq \frac{g(0)+g(x)}{2}
$$

Consecuentemente se obtiene

$$
\frac{f^{p}(0)+f^{p}(x)}{2}\left(\mathcal{J}_{\rho, \lambda_{2}, 0+; w}^{\sigma} x^{\lambda_{1}-1}\right) \geq\left(\frac{f(0)+f(x)}{2}\right)^{p}\left(\mathcal{J}_{\rho, \lambda_{2}, 0+; w}^{\sigma} x^{\lambda_{1}-1}\right)
$$

$y$

$$
\frac{g^{q}(0)+g^{q}(x)}{2}\left(\mathcal{J}_{\rho, \lambda_{2}, 0+; w}^{\sigma} x^{\lambda_{1}-1}\right) \geq\left(\frac{g(0)+g(x)}{2}\right)^{q}\left(\mathcal{J}_{\rho, \lambda_{2}, 0+; w}^{\sigma} x^{\lambda_{1}-1}\right) .
$$

De estas últimas desigualdades se tiene que

$$
\begin{gathered}
\frac{\left(f^{p}(0)+f^{p}(x)\right)\left(g^{q}(0)+g^{q}(x)\right)}{4}\left(\mathcal{J}_{\rho, \lambda_{2}, 0+; w}^{\sigma} x^{\lambda_{1}-1}\right)^{2} \\
\geq 2^{-p-q}(f(0)+f(x))^{p}(g(0)+g(x))^{q}\left(\mathcal{J}_{\rho, \lambda_{2}, 0+; w}^{\sigma} x^{\lambda_{1}-1}\right)^{2} .
\end{gathered}
$$

Combinando las desigualdades (3.10) y (3.11) se obtiene la desigualdad buscada.

Observación 3. Si $\lambda_{1}=\lambda_{2}=\lambda$ en el Teorema 3 entonces se obtiene que

$$
\begin{aligned}
& 2^{-p-q}(f(0)+f(x))^{p}(g(0)+g(x))^{q}\left(\mathcal{J}_{\rho, \lambda, 0+; w}^{\sigma}\left(x^{\lambda-1}\right)\right)^{2} \\
& \leq\left(\mathcal{J}_{\rho, \lambda, 0+; w}^{\sigma}\left(x^{\lambda-1} f^{p}\right)\right)(x)\left(\mathcal{J}_{\rho, \lambda, 0+; w}^{\sigma}\left(x^{\lambda-1} g^{q}\right)\right)(x),
\end{aligned}
$$

y por consiguiente, si $\lambda=\alpha, \sigma=(1,0,0, \ldots)$ y $w=0$ se obtiene la siguiente desigualdad

$$
2^{-p-q}(f(0)+f(x))^{p}(g(0)+g(x))^{q}\left(I_{0+}^{\alpha} x^{\lambda-1}\right)^{2} \leq\left(I_{0+}^{\alpha} x^{\lambda-1} f^{p}\right)(x)\left(I_{0+}^{\alpha} x^{\lambda-1} g^{q}\right)(x)
$$

la cual coincide con el Teorema 2.5 in [6]. En particular, si $\alpha=1$ entonces

$$
2^{-p-q}(f(0)+f(x))^{p}(g(0)+g(x))^{q} \leq \frac{1}{(b-a)^{2}}\|f\|_{p}^{p}\|g\|_{p}^{p}
$$

con lo cual se encuentra el Teorema 2.3 en [7].

Observación 4. Haciendo $\lambda_{1}=\beta, \lambda_{2}=\alpha, \sigma=(1,0,0, \ldots)$ y $w=0$ en el Teorema 3 se tiene que

$$
2^{-p-q}(f(0)+f(x))^{p}(g(0)+g(x))^{q}\left(I_{0+}^{\alpha} x^{\beta-1}\right)^{2} \leq\left(I_{0+}^{\alpha} x^{\beta-1} f^{p}\right)(x)\left(I_{0+}^{\alpha} x^{\beta-1} g^{q}\right)(x)
$$

lo cual hace coincidencia con el Teorema 2.8 in [6]. Si $\alpha=\beta$ entonces se tiene el Teorema 2.5 in [6].

En la demostración del próximo Teorema se hará uso de la desigualdad de Young:

$$
x y \leq \frac{x^{p}}{p}+\frac{y^{q}}{q}, \text { para cualesquiera } x, y \geq 0 \text { y } p>1, \frac{1}{p}+\frac{1}{q}=1 .
$$

Teorema 4. Sean $p, q \in \mathbb{R}$ con $p>1$ y $1 / p+1 / q=1$. Sean $f, g: a, b \rightarrow \mathbb{R}$ funciones tales que $f^{p}, g^{q}, f g \in$ $L_{1}([a, b]) y$

$$
0<m \leq \frac{f(x)}{g(x)} \leq M, \text { para cualesquiera } x \in[a, b], a, b \in[0, \infty)
$$


entonces

$$
\begin{aligned}
\left(\mathcal{J}_{\rho, \lambda, a+; w}^{\sigma} f g\right)(x) \leq \frac{2^{p-1}}{p} & \left(\frac{M}{M+1}\right)^{p}\left(\left(\mathcal{J}_{\rho, \lambda, a+; w}^{\sigma} f^{p}\right)(x)+\left(\mathcal{J}_{\rho, \lambda, a+; w}^{\sigma} g^{p}\right)(x)\right) \\
& +\frac{2^{q-1}}{q}\left(\frac{1}{m+1}\right)^{q} 2^{q-1}\left(\left(\mathcal{J}_{\rho, \lambda, a+; w}^{\sigma} f^{q}\right)(x)+\left(\mathcal{J}_{\rho, \lambda, a+; w}^{\sigma} g^{q}\right)(x)\right) .
\end{aligned}
$$

Demostración: Del hecho que $0<m \leq f(x) / g(x) \leq M$, para cualquier $x \in[a, b]$, se tiene que

$$
\begin{aligned}
& f(x) \leq \frac{M}{M+1}(f(x)+g(x)), \\
& g(x) \leq \frac{1}{m+1}(f(x)+g(x)) .
\end{aligned}
$$

Usando la desigualdad de Young

$$
f(x) g(x) \leq \frac{f(x)^{p}}{p}+\frac{g(x)^{q}}{q} \leq \frac{1}{p}(f(x)+g(x))^{p}+\frac{1}{q}(f(x)+g(x))^{q}
$$

entonces, multiplicando por $(x-t)^{\lambda-1} \mathcal{F}_{\rho, \lambda}^{\sigma}\left[w(x-t)^{\rho}\right]$

$$
\begin{aligned}
\int_{a}^{x}(x-t)^{\lambda-1} \mathcal{F}_{\rho, \lambda}^{\sigma}\left[w(x-t)^{\rho}\right] f(t) g(t) d t \\
\leq \frac{1}{p}\left(\frac{M}{M+1}\right)^{p} \int_{a}^{x}(x-t)^{\lambda-1} \mathcal{F}_{\rho, \lambda}^{\sigma}\left[w(x-t)^{\rho}\right]\left(f(t)+g(t)^{p} d t\right. \\
\quad+\frac{1}{q}\left(\frac{1}{m+1}\right)^{q} \int_{a}^{x}(x-t)^{\lambda-1} \mathcal{F}_{\rho, \lambda}^{\sigma}\left[w(x-t)^{\rho}\right](f(t)+g(t))^{q} d t
\end{aligned}
$$

Usando la desigualdad clásica: $(c+d)^{p} \leq 2^{p-1}\left(c^{p}+d^{p}\right)$, se obtiene

$$
\begin{aligned}
& \int_{0}^{x}(x-t)^{\lambda-1} \mathcal{F}_{\rho, \lambda}^{\sigma}\left[w(x-t)^{\rho}\right] f(t) g(t) d t \\
& \leq \frac{1}{p}\left(\frac{M}{M+1}\right)^{p} 2^{p-1} \int_{a}^{x}(x-t)^{\lambda-1} \mathcal{F}_{\rho, \lambda}^{\sigma}\left[w(x-t)^{\rho}\right]\left(f^{p}(t)+g^{p}(t)\right) d t \\
& +\frac{1}{q}\left(\frac{1}{m+1}\right)^{q} 2^{q-1} \int_{a}^{x}(x-t)^{\lambda-1} \mathcal{F}_{\rho, \lambda}^{\sigma}\left[w(x-t)^{\rho}\right]\left(f^{q}(t)+g^{q}(t)\right) d t \\
& =\frac{1}{p}\left(\frac{M}{M+1}\right)^{p} 2^{p-1}\left(\left(\mathcal{J}_{\rho, \lambda, a+; w}^{\sigma} f^{p}\right)(x)+\left(\mathcal{J}_{\rho, \lambda, a+; w}^{\sigma} g^{p}\right)(x)\right) \\
& +\frac{1}{q}\left(\frac{1}{m+1}\right)^{q} 2^{q-1}\left(\left(\mathcal{J}_{\rho, \lambda, a+; w}^{\sigma} f^{q}\right)(x)+\left(\mathcal{J}_{\rho, \lambda, a+; w}^{\sigma} g^{q}\right)(x)\right) .
\end{aligned}
$$

La prueba está completada.

\section{Observación 5.}

Haciendo $\lambda=\alpha, w=0$ y $\sigma=(1,0,0, \ldots)$ en el Teorema 4 se obtiene la desigualdad para la integral fraccionaria de Riemann-Liouville

$$
\begin{aligned}
\left(I_{a+}^{\alpha} f g\right)(x) \leq & \frac{2^{p-1}}{p}\left(\frac{M}{M+1}\right)^{p}\left(\left(I_{0+}^{\alpha} f^{p}\right)(x)+\left(I_{0+}^{\alpha} g^{p}\right)(x)\right) \\
& +\frac{2^{q-1}}{q}\left(\frac{1}{m+1}\right)^{q}\left(\left(I_{0+}^{\alpha} f^{q}\right)(x)+\left(I_{0+}^{\alpha} g^{q}\right)(x)\right) .
\end{aligned}
$$

En particular, si $\alpha=1$ se tiene

$$
\int_{0}^{x} f(t) g(t) d t \leq \frac{2^{p-1}}{p}\left(\frac{M}{M+1}\right)\left(\|f\|_{p}^{p}+\|g\|_{p}^{p}\right)+\frac{2^{q-1}}{q}\left(\frac{1}{m+1}\right)^{q}\left(\|f\|_{q}^{q}+\|g\|_{q}^{q}\right) .
$$

haciendo coincidencia con el Teorema 2.4 en [7]. 


\section{Conclusiones.}

En el presente artículo se establecieron algunas desigualdades integrales fraccionarias del tipo Minkowski y Hermite-Hadamard (Teoremas 1,2,3,4 y 5) haciendo uso del operador integral fraccionario introducido por R.K. Raina en [1]. Además se presentaron algunas observaciones conducentes a establecer la generalización de los resultados obtenidos con respecto a otros previamente encontrados por L. Bougoffa [5] y S.S. Dragomir [7]. Es de hacer notar que se pueden generalizar los resultados obtenidos usando la siguiente integral fraccional definida por T. Tunç et.al. en [19]:

$$
\left(\mathcal{J}_{\rho, \lambda, a+; w}^{\sigma, k, g} \varphi\right)(x)=\int_{a}^{x} \frac{g^{\prime}(t)}{\left((g(x)-g(t))^{1-\frac{\lambda}{k}}\right.} \mathcal{F}_{\rho, \lambda}^{\sigma, k}\left[w(x-t)^{\rho}\right] \varphi(t) d t, \quad(x>a)
$$

$y$

$$
\left(\mathcal{J}_{\rho, \lambda, b-; w}^{\sigma, k, g} \varphi\right)(x)=\int_{x}^{b} \frac{g^{\prime}(t)}{\left((g(x)-g(t))^{1-\frac{\lambda}{k}}\right.} \mathcal{F}_{\rho, \lambda}^{\sigma, k}\left[w(t-x)^{\rho}\right] \varphi(t) d t, \quad(x<b),
$$

donde

$$
\mathcal{F}_{\rho, \lambda}^{\sigma, k}(x)=\sum_{m=0}^{\infty} \frac{\sigma(m)}{k \Gamma_{k}(\rho k m+\lambda)} x^{m}
$$

$k>0, g:[a, b] \rightarrow \mathbb{R}$ es una función positiva y creciente con derivada $g^{\prime}$ es continua sobre $(a, b), \rho, \lambda>0,|x|<\mathbb{R}$ y $\sigma=(\sigma(1), . ., \sigma(k), .$.$) es una sucesión acotada de números reales, \Gamma_{k}$ es la función $k-G a m m a, y$ es una función tal que las integrales expuestas existen.

El autor espera que los resultados encontrados sirvan de estímulo para futuras investigaciones en el área.

Agradecimientos. El autor agradece al Consejo para el Desarrollo de las Ciencias, Humanidades y Tecnología (CDCHT) de la Universidad Centroccidental Lisandro Alvarado (Venezuela) por el soporte tecnológico brindado en la elaboración de este artículo. De la misma forma, agradece al Dr. Miguel José Vivas-Cortez por sus valiosos aportes y comentarios. Igualmente agradece a los árbitros asignados por la Revista Selecciones Matemáticas de La Universidad de Trujillo (Perú) por la colaboración prestada en la evaluación del presente artículo.

\section{Referencias}

[1] Agarwal, R. P., Luo, M-J. \& Raina, R. K; On Ostrowski Type Inequalities, Fasculli Mathematici. 2016 ; 56 : 5 - 27.

[2] Beckenbach, E. \& Bellman, R.; An Introduction to inequalities, L.W. Silver Company, 1961

[3] Beckenbach, E. \& Bellman, R.; Inequalities, Springer-Verlag, Berlin, 1961

[4] Belarbi, S. \& Dahmani, Z.; On some new fractional integral inequalities. Journal on Inequalities in Pure and Applied Mathematics; 2009, 10(3): 1 - 5. pp

[5] Bougoffa, L.; On Minkowski and Hardy Integral Inequalities. Jounal of Inequalities in Pure and Applied Mathematics. 2006; 7(2).

[6] Dahmani, Z.; On Minkowski and Hermite-Hadamard Integral Inequalities Via Fractional Integration. Annals of Functional Analysis; 2010, 1(1): $51-58$

[7] Dragomir, S. S., Set, E. \& Özdemir, M. E.; On the Hermite-Hadamard Inequality and Other Integral Inequalities Involving Two Functions. Journal of Inequalities and Applications; 2010, Article ID 148102: 1 - 9.

[8] Gorenflo, R. \& Mainardi, F.; Fractional Calculus: Integral and Differential Equations of Fractional Order. CISM Courses and Lect. , Springer, 378: 223 - 276.

[9] Hadamard, J. Etude sur les proprietes des fonctions entieres et en particulier d'une fonction considree par Riemann. Journal de Mathématiques pur et appliqué; 1893, 58(1): 171- 215.

[10] Hardy, G. H., Littlewood, J. E. \& Pólya, G.; Inequalities, Cambridge Press., London, 1934

[11] Hermite, Ch.; Sur deux limites d'une integrale definie. Mathesis; 1883, 3(1): 1 -82.

[12] Iqbal, M., Iqbal, B. M. \& Nazeer, K.; Generalization of Inequalities Analogous to Hermite-Hadamard Inequality via Fractional Integrals. Bulletin of the Korean Mathematical Society, 2015, 523:707 - 716

[13] Marinkovic, S., Rajkovic, P. \& Stankovic, M.; The inequalities for some types q-integrals. Computers and Mathematics with Applications; 2008, 56: 2490 - 2498.

[14] Marshall, A. W. \& Olkin, I.; Inequalities: Theory of Majoration and Applications, Academic Press, 1979.

[15] Miller, S. \& Ross, B. An introduction to the Fractional Calculus and Fractional Differential Equations. 1993, John Wiley and Sons, USA.

[16] Niculescu, C. P. \& Persson, L. E. Convex functions and their applications, A comtemporary approach. CMS Books in Mathematics, 23, Springer Verlag, New York, 2006

[17] Raina, R.K.; On Generalized Wright's Hypergeometric Functions and Fractional Calculus Operators. East Asian Mathematical Journal; 2005, 21(2): 191 - 203. 191-203

[18] Sarikaya, M. Z., Set, E., Yaldiz, H. \& Basak, N.; Hermite- Hadamard's inequalities for fractional integrals and related fractional inequalities. Mathematical and Computer Modelling, 2017, 57:2403 - 2407

[19] Tunç, T. , Budak, H., Usta, F. \& Sarikaya, M. Z.; On new generalized fractional integral operators and related inequalities. Submitted article, ResearchGate. https://www.researchgate.net/publication/313650587 [12 November 2018 